\title{
First report on molecular evidence of Candidatus Liberibacter asiaticus associated with citrus Huanglongbing in Venezuela
}

\author{
Edgloris Marys ${ }^{1}$ (1) Eduardo Rodríguez-Román ${ }^{1}$ (1) $\cdot$ Rafael Mejías $^{2} \cdot$ Alexander Mejías $^{1} \cdot$ Mailyn Mago $^{3}$. \\ Yonis Hernández ${ }^{2}$
}

Received: 10 March 2020 / Accepted: 14 July 2020 / Published online: 21 July 2020

(C) Società Italiana di Patologia Vegetale (S.I.Pa.V.) 2020

Keywords Huanglongbing $\cdot$ PCR $\cdot$ Detection $\cdot$ Venezuela $\cdot$ Candidatus Liberibacter asiaticus

Huanglongbing disease (citrus greening; HLB) is currently the most economically damaging disease of citrus trees, and has been associated with the collapse of the several citrus industries in Asia and America. The disease is associated with the presence of a phloem restricted alpha proteobacteria species of the 'Candidatus Liberibacter' genus. 'Candidatus Liberibacter asiaticus' (CLas) is the dominant species found in South America (Lopes et al. 2009). In 2017, branches were collected from Citrus sp. trees showing mild to severe yellowing on the shoots, lopsided and greening fruits and tree decline in various regions in Venezuela. To verify the presence of CLas, total DNA was extracted from single leaf midribs and subjected to PCR amplification with primers sets OI1/OI2c targeting the $16 \mathrm{~S}$ rDNA locus (Jagoueix et al. 1994) and A2/J5 targeting the beta-operon locus of ribosomal proteins (Hocquellet et al. 1999). A total of 87 out of 104 samples (83.7\%) tested positive with both primer sets. The OI1/OI2c primers generated an amplicon of approximately $1160 \mathrm{bp}$ and digestion by XbaI yielded two DNA fragments of approximately $640 \mathrm{bp}$ and 520 bp, suggesting the presence of CLas (Jagoueix et al. 1994). The A2/J5 primer set generated a 703-bp amplicon, suggesting 'Candidatus L. africanus' was not present. Two of the A2/J5 amplicons were purified, cloned and sequenced. The derived sequences have been deposited in the GenBank database under accession Nos. MG418842 and MG418841. Sequence comparison revealed that MG418842 and MG418841 shared $>99 \%$ identity to the corresponding regions of CLas in the GenBank database. To our knowledge, this is the first report of molecular identification of CLas infecting citrus in Venezuela.

\section{References}

Hocquellet A, Toorawa P, Bové JM, Garnierf M (1999) Detection and identification of the two Candidatus Liberobacter species associated with citrus huanglongbing by PCR amplification of ribosomal protein genes of the $\beta$ operon. Mol Cell Probes 13:373-379

Jagoueix S, Bove JM, Garnier M (1994) The phloem-limited bacterium of greening disease of citrus is a member of the a subdivision of the Proteobacteria. Int J Syst Evol Microbiol 44:379-386

Lopes SA, Bertolini E, Frare GF, Martins EC, Wulff NA et al (2009) Graft transmission efficiencies and multiplication of 'Candidatus Liberibacter americanus' and ' $\mathrm{Ca}$. Liberibacter asiaticus' in citrus plants. Phytopathology 99:301-306

Publisher's note Springer Nature remains neutral with regard to jurisdictional claims in published maps and institutional affiliations.

Edgloris Marys

edgloris@gmail.com

1 Laboratorio de Biotecnología y Virología Vegetal, Centro de Microbiología y Biología Celular, Instituto Venezolano de Investigaciones Científicas (IVIC), ZIP 20632, Caracas 1020-A, Venezuela

2 Facultad de Agronomía, Laboratorio de Bacterias Fitopatógenas, Universidad Central de Venezuela (UCV), Caracas, Venezuela 